\title{
Effect of diamagnetic contribution of water on harmonics distribution in a dilute solution of iron oxide nanoparticles measured using high- $T_{\mathrm{c}}$ SQUID magnetometer
}

\author{
Mohd Mawardi Saari ${ }^{1}$, Yuya Tsukamoto, Toki Kusaka, Yuichi Ishihara, Kenji Sakai, Toshihiko \\ Kiwa and Keiji Tsukada
}

Graduate School of Natural Science and Technology, Okayama University, 3-1-1 Tsushima Naka, Kita Ku, Okayama 700-8530, JAPAN

\begin{abstract}
The magnetization curve of iron oxide nanoparticles in low-concentration solutions was investigated by a highly sensitive high- $T_{\mathrm{c}}$ superconducting quantum interference device (SQUID) magnetometer. The diamagnetic contribution of water that was used as the carrier liquid was observed in the measured magnetization curves in the high magnetic field region over $100 \mathrm{mT}$. The effect of the diamagnetic contribution of water on the generation of harmonics during the application of AC and DC magnetic fields was simulated on the basis of measured magnetization curves. Although the diamagnetic effect depends on concentration, a linear relation was observed between the detected harmonics and concentration in the simulated and measured results. The simulation results suggested that improvement could be expected in harmonics generation because of the diamagnetic effect when the iron concentration was lower than $72 \mu \mathrm{g} / \mathrm{ml}$. The use of second
\end{abstract}

\footnotetext{
${ }^{1}$ Author to whom correspondence should be addressed. Electronic mail : en19463@s.okayama-u.ac.jp

Phone : +81-086-251-8129
} 
harmonics with an appropriate bias of the DC magnetic field could be utilized for realization of a fast and highly sensitive detection of magnetic nanoparticles in a low-concentration solution.

\section{Keywords}

magnetic nanoparticles, water, harmonics generation, SQUID magnetometer 


\section{Introduction}

Magnetic nanoparticles have been utilized in medical imaging as contrast agents and tracers [1]. In addition, the use of such nanoparticles in bio-immunoassay, magnetic nanoparticle imaging, and magnetic drug targeting has been studied owing to their promising results in these applications. The inherent magnetic properties of these particles can be determined by measuring their magnetic susceptibility [2-4], relaxation [5-7], and remanence [8,9]. Lately, some researchers have reported the development of sensitive measurement systems that are capable of measuring the magnetic relaxation and remanence of magnetic nanoparticles in solution [7,10,11]. Although the magnetic responses of magnetic nanoparticles in solutions of different concentrations have been widely studied by observing magnetic relaxation, remanence, and AC susceptibility, the behavior of a lowconcentration solution of magnetic nanoparticles in wide magnetic field regions still remains unclear. This might be because conventional magnetometers such as low-sensitivity magnetic sensors have certain limitations and the magnetic properties are often measured using concentrated and/or powdered samples. Furthermore, the magnetic response of the nanoparticles is assumed to be similar in solutions of different concentrations and the effect of the diamagnetic carrier liquid is neglected even in diluted solutions. This requires clarification, as most biomedical applications involve measurements of magnetic nanoparticles in low-concentration solutions, wherein the diamagnetic background signal from the carrier liquid may be comparable to the magnetic responses of the nanoparticles. Therefore, development of highly sensitive magnetometers is critical in order to detect small amounts of magnetic nanoparticles in low concentration solutions. Furthermore, magnetic susceptibility must be measured in the presence of an excitation magnetic field, in contrast to the measurement of magnetic relaxation and remanence. Interferences from the excitation magnetic field may limit the sensitivity of measurement systems, particularly in the case of AC susceptometer systems [12-14]. Such interferences from the excitation magnetic fields 
during dynamic magnetization measurements have been reduced by employing harmonics generated from the nonlinear magnetization characteristics of MNPs [15-17]. These harmonics are used to quantify MNPs with fast measurements and improve their sensitivity by isolating the frequency component of excitation magnetic fields. In addition, large amplitudes of excitation magnetic fields can further lead to enhancement in the generation of harmonics, as they tend to cover the wider regions of the nonlinear characteristics.

In this study, we have developed a highly sensitive AC-DC magnetometer using a highcritical-temperature superconducting quantum interference device (high- $T_{\mathrm{c}}$ SQUID) on the basis of our previously developed system $[18,19]$. This flux transformer-based high- $T_{c}$ SQUID exhibited high sensitivity with less interference from the excitation magnetic fields. Using the developed system, we measured the static magnetizations and harmonics distribution of low-concentration solutions of iron oxide nanoparticles and investigated their magnetic responses in solutions of different concentrations. As a preliminary analysis to investigate the relationship between the concentration and the harmonics generation of diluted iron oxide nanoparticles during the application of DC and AC magnetic fields, we simulated the generation of harmonics on the basis of measurement results of the static magnetization. The static magnetization curves indicate the contribution of the diamagnetism of water as a function of concentration. Detection of lowconcentration magnetic nanoparticles with high sensitivity has been demonstrated using second harmonics. 


\section{Material and method}

\subsection{Iron oxide nanoparticles}

Iron oxide nanoparticles analyzed in this study were nanomag®-D-spio (Micromod Partikeltechnologie GmbH, Rostock-Warnemuende, Germany). In the typical experiment, a predetermined quantity of iron oxide nanoparticles was suspended in water such that the concentration of iron in the resulting solution is $2.4 \mathrm{mg} / \mathrm{ml}$. The iron oxide nanoparticles used in this study have an overall diameter of $100 \mathrm{~nm}$, and consist of dextran iron oxide composites. Subsequently, lowconcentration solutions were prepared by further diluting the suspension in purified water to obtain iron concentrations of $24 \mu \mathrm{g} / \mathrm{ml}, 48 \mu \mathrm{g} / \mathrm{ml}, 72 \mu \mathrm{g} / \mathrm{ml}$, and $96 \mu \mathrm{g} / \mathrm{ml}$. The diluted iron oxide solutions thus obtained were stored in 3-ml acrylic cases for further analysis.

\subsection{AC-DC high- $T_{\mathrm{c}}$ SQUID magnetometer for evaluating magnetic nanoparticles in solution}

Fig.1 illustrates the overview of the high- $T_{\mathrm{c}}$ SQUID developed in this study and the coil arrangements used for static and dynamic magnetization measurements. The flux transformer consists of first-order planar and axial differential coils as the pickup coils for static and dynamic magnetization measurements, respectively, to reduce the environmental noise being transferred to the high- $T_{\mathrm{c}}$ SQUID. Both types of coils were constructed by connecting two identical elliptical coils in a series opposing configuration. A ramp-edge-type Josephson junction was fabricated on an $\mathrm{MgO}$ substrate by a multilayer fabrication technique [20]. The sensitivity of the magnetic susceptibility of the developed system was $1 \times 10^{-8} \mathrm{emu}$ (dimensionless). Details of the measurement system have been reported elsewhere $[18,19]$.

For static magnetization measurements, the sample was exposed to a DC magnetic field generated by an electromagnet and perpendicularly vibrated to the axis of the DC magnetic field 
using an actuator having amplitude $5 \mathrm{~mm}$ and vibration frequency $2.82 \mathrm{~Hz}$. The sensitive axis of the planar differential coil was maintained parallel to the axis of the DC magnetic field, as shown in Fig. 1 (a). The induced signals were transferred to the inductively coupled SQUID and the output was detected by a lock-in amplifier. The motion of the actuator that was detected by a laser position sensor was used as a reference signal for the lock-in amplifier. The magnetization curve of the diluted solutions was determined in the range from -260 to $260 \mathrm{mT}$ with a complete cycle of magnetization loop.

The characteristics and the concentration of the iron oxide nanoparticles in the solution could be quantitatively determined from the static magnetization curve; however, this is a time consuming process. Some biomedical applications such as bio-immunoassay require simultaneous measurements of multiple samples. For such applications, harmonics detection [15,21] can provide fast information on the magnetic response of magnetic nanoparticles by covering wide magnetic field regions in one-shot measurements. In this technique, the distribution of the induced harmonics of magnetic nanoparticles is related to the applied AC and DC magnetic field bias and the magnetization curve. For an AC magnetic field with amplitude larger than saturation magnetic field $H_{\mathrm{s}}$, the magnetic response consists of harmonic components in the region below $H_{\mathrm{s}}$, whereas in the region above $H_{\mathrm{s}}$, the magnetic response is suppressed because of moment saturation. However, the introduction of the DC magnetic field will result in the magnetic response of the magnetic nanoparticles following the magnetization curve and production of a distorted waveform of the magnetic field with odd and even numbers of harmonic components [16,17]. Cutting the fundamental frequency component with a filter and measuring the harmonic components of the magnetic response will result in a high signal-to-noise ratio. In our measurement system, the sensitive axis of the axial differential coil was placed perpendicular to the excitation magnetic field so as to reduce the interference of the excitation magnetic field. As shown in Fig. 1 (b), the sample 
was placed at the center of the axial differential coil to which an AC magnetic field of frequency 5 $\mathrm{Hz}$ superimposed with a DC magnetic field was applied. The high- $T_{\mathrm{c}}$ SQUID output was detected by a lock-in amplifier using a reference signal at a function generator. The excitation magnetic fields were determined by a Hall sensor. Second and third harmonics were measured with different AC amplitudes and DC biases of magnetic fields to investigate their distributions and the effect of low concentration on magnetic response.

\section{Theoretical model}

\subsection{Static magnetization model}

The magnetization curves of the magnetic nanoparticles can be described by the Langevin function under the assumption that they are not affected by interparticle interactions and that they have isotropic spin governed by thermal fluctuations and the magnetization field [16,22]. This model is applicable to the iron oxide nanoparticles analyzed in this study as they were well dispersed in the diluted suspensions. Although the size of the iron oxide nanoparticles was distributed to some extent, which could be represented by the so-called log-normal spread [23], to avoid complexity during the fitting procedure, a mean diameter was assumed for the iron oxide nanoparticles. Moreover, since the iron concentration in the solution was low, the diamagnetic signal from the carrier liquid might be significant enough to decrease the magnetization curve in high magnetic field regions. This effect was corrected by introducing the parameter $C$. Accordingly, the magnetization curve of the diluted iron oxide nanoparticles can be expressed as

$$
M\left(\mu_{0} H\right)=M_{S} L\left(\frac{m \mu_{0} H}{k_{B} T}\right)-C \mu_{0} H
$$


where $L\left(m \mu_{0} H / k_{B} T\right)$ is the Langevin function, $m$ is the magnetic moment of iron oxide nanoparticles, and $k_{\mathrm{B}}$ is the Boltzmann constant. Furthermore, $M_{S}=\phi M_{0}$ is the saturation magnetization, which is the product of the volume concentration $\phi$ of the suspended magnetic nanoparticles and their spontaneous magnetization $M_{0}$. In this model, we treated $M_{\mathrm{S}}, C$, and $m$ as functions of concentration in order to fit with the static magnetization data by Mathematica (Wolfram Research, Champaign, IL, USA) using the least-squares fitting method.

\subsection{Harmonics generation}

In a diluted solution, the addition of the diamagnetic signal of the carrier liquid due to the deformation of the magnetization curve might affect harmonics generation. For a low frequency of the AC magnetic field, the time varying magnetization can be assumed to follow the magnetization curve. Magnetization $M(\mathrm{t})$ resulting from the excitation of magnetic field $B_{\text {excitation }}(t)=B_{\text {amp }} \sin (\omega t)+$ $B_{D C}$ can be expressed as

$$
\begin{aligned}
M(t) & =M\left(B_{\text {exitation }}(t)\right) \\
& =M_{S} L\left(\frac{m B_{\text {exitation }}(t)}{k_{B} T}\right)-C B_{\text {exitation }}(t) \\
& =M_{0}^{\prime}+M_{1}^{\prime} \cos \left(\omega t+\theta_{1}\right)+M_{2}^{\prime} \cos \left(2 \omega t+\theta_{2}\right)+\cdots+M_{n}^{\prime} \cos \left(n \omega t+\theta_{n}\right)
\end{aligned}
$$

where $M_{n}^{\prime}$ is the amplitude coefficient and $\theta_{n}$ is the phase angle at angular frequency $n \omega$. The signal response detected by a normal conductive coil can be calculated by the time derivative of $M(t)$. In addition, we performed Fast Fourier Transform (FFT) analysis to extract harmonic components. The fundamental, second, and third harmonics were analyzed, as the higher harmonic components rapidly decrease with increasing harmonic number. 


\section{Magnetization curve of iron oxide nanoparticles solution}

Fig. 2 shows the measured magnetization curves of the iron oxide nanoparticles in different diluted solutions after subtraction of the signal from the acrylic cases. The magnetization curve of purified water used in this study was included as a reference. The magnetization curves show superparamagnetic characteristics with $H_{\mathrm{s}}$ of approximately $\mu_{0} H=40 \mathrm{mT}$, where $\mu_{0}$ is the vacuum permeability and $H$ is the magnetic field strength. Interestingly, for a low-concentration solution of the magnetic nanoparticles, the magnetization curve did not show saturation but showed a diamagnetic effect in the high magnetic field region.

The measured results were quantitatively analyzed by fitting the data using Eq. (1). The fitted graphs are indicated by dashed lines in Fig. 2, and the fitted parameters are denoted in Fig. 3. $M_{\mathrm{S}}$ almost linearly corresponds to the increasing concentrations, whereas $C$ rapidly decreases with concentration. This suggests that the diamagnetic signal of the carrier liquid in a dilute solution of magnetic nanoparticles should not be completely ignored, because the diamagnetism of the solution might exist as a function of concentration. As the concentration of iron oxide nanoparticles increases in high concentration solutions, the diamagnetic signal of water can be predicted to linearly decrease with decreasing volume percentage of water. However, the substantial decrease in $C$ with increasing concentration could be attributed to the increasing number of small as well as large particles at higher concentrations. The large particles saturate and increase the saturation magnetization at higher concentrations. However, the small particles do not saturate even in a high magnetic field [24], thus canceling out the diamagnetic signal of water. Therefore, the diamagnetic effect rapidly disappeared at higher concentrations. The values of $m$ were similar for solutions of different concentrations in the range from 3.3 to $3.4 \times 10^{-17} \mathrm{Am}^{2}$. This value indicates the size distribution of the iron oxide nanoparticles was similar at all concentrations. Further studies using 
fine particles could improve our understanding from this viewpoint. Nonetheless, the results show that the diamagnetic contribution of the carrier liquid has an effect on magnetization curves, when the magnetic moments of iron oxide nanoparticles and water are compared. This behavior could be beneficial in harmonics detection.

\section{Simulation of harmonics generation}

The deformation of the magnetization curve due to diamagnetic effect can be utilized in the harmonics generation method to increase the signal-to-noise ratio in a low concentration solution of iron oxide nanoparticles. The magnetic response due to the application of AC and DC magnetic fields may consist of a high ratio of odd and even numbers of harmonic components. As a preliminary investigation, we simulated the magnetic responses of the measured magnetization curves using the fitted parameters. The distribution of the fundamental, second, and third harmonics with respect to the DC magnetic field during excitation of AC magnetic field of amplitude $30 \mathrm{mT}$ for different concentrations are displayed in Fig. 4. At the offset values of the DC magnetic field, where the signals were at a maximum, the harmonic components almost linearly increased with increasing concentrations, as indicated in the superimposed graphs. The fundamental component increased with decreasing concentration in the high DC magnetic field region due to increments in the diamagnetic effect of the carrier liquid. The signal intensity of the second harmonic component was comparable with that of the fundamental component. Therefore, compared with the third harmonic component, the second harmonic component could be utilized for quantitative analysis of the iron oxide nanoparticles by biasing an appropriate DC magnetic field. Moreover, as is evident in Fig. 5, the ratio of the harmonic components to the fundamental component at their maximum points in Fig. 4 was considerably improved for the low concentration solutions. Deformation of the 
magnetization curve due to the diamagnetism of water might be advantageous for the measurement of a low concentration solution, where the fundamental harmonic signal is subject to interference by the excitation magnetic field. In addition, a high signal-to-noise ratio of the signal can still be obtained using second harmonics. More importantly, the diamagnetic effect further enhanced harmonics generation using the large amplitude AC magnetic field. Furthermore, their responses with respect to the DC magnetic field were similar compared with the case of the small amplitude AC magnetic field. This preliminary result demonstrates the feasibility of fast analysis of magnetic nanoparticles in solutions by detecting the harmonic components with an appropriate bias of the DC magnetic field.

\section{Harmonics signal of iron oxide nanoparticles solution}

In an attempt to compensate for circumference noise, the signals detected from the lock-in amplifier with and without the samples were subtracted during harmonics measurements. Because of substantial interference from the residual excitation magnetic field, which was equivalent to 76 $\mu \mathrm{T}(700 \mathrm{mV})$, the fundamental component could not be measured. The magnetic response of the diluted solutions was estimated to be a few tens of nT. Fig. 6 illustrates the distribution of the second and third harmonics of the $24-\mu \mathrm{g} / \mathrm{ml}$ iron oxide nanoparticles solution with respect to the different AC magnetic field amplitudes biased with the DC magnetic fields from 0 to $100 \mathrm{mT}$. In the case where diamagnetic effect was the largest, a superimposed contour map was presented as a comparison for the simulation results discussed in Section 5. The signals of the second and third harmonics increased with larger AC magnetic field amplitudes and an appropriate bias of the DC magnetic field. The position and distribution of the peaks were consistent with the simulation results. The response of the second harmonics revealed less noise compared with that of the third 
harmonics, suggesting an additional advantage of using second harmonics in our measurement system. Fig. 7 shows the second and third harmonics corresponding to the DC magnetic field at 20 and $0 \mathrm{mT}$, respectively, during the excitation of the AC magnetic field with an amplitude of $30 \mathrm{mT}$ with respect to the concentration. The second and third harmonics linearly responded to increasing concentrations, consistent with simulated results shown in the superimposed graphs in Fig. 4. This proves the existence of a linear relation between detected harmonics and concentration, despite the dependence of diamagnetic effect on the concentration. However, as the fundamental component was completely buried in the residual magnetic field, the effect of the diamagnetic contribution of water on the ratio of the harmonics could not be directly assessed.

\section{Conclusion}

In this study, we developed a high- $T_{\mathrm{c}}$ SQUID magnetometer and utilized it to investigate the magnetization curve and harmonics distributions of low-concentration solutions of iron oxide nanoparticles. The static magnetization results of the low-concentration solutions showed that the diamagnetic signal from the carrier liquid deformed the magnetization curve and the diamagnetism of the solutions existed as a function of concentration. This suggests that the diamagnetic effect in the high magnetic field region could not be ignored while measuring a diluted solution of magnetic nanoparticles, for instance in biomedical applications. To facilitate fast analysis of magnetic nanoparticles, we simulated the generation of harmonics on the basis of measurement results of the static magnetization. The simulation results reveal that, at an appropriate bias of the DC magnetic field, the harmonic components linearly increase with increasing concentration, in the existence of diamagnetism of carrier liquid. Furthermore, the diamagnetic effect of the carrier liquid was beneficial for the harmonics detection technique from the perspective that the ratio of the harmonics

to fundamental components had considerably improved. In the harmonics measurement, a linear 
relation between harmonics and concentration was obtained at an appropriate bias of the DC magnetic field. The harmonics distributions were similar to the simulated data. The use of second harmonics with an appropriate bias of the DC magnetic field showed that a high signal-to-noise ratio of measurement could be obtained when measuring dilute solutions of magnetic nanoparticles in the large interference of the excitation magnetic field.

\section{Acknowledgement}

This work is supported by the "Strategic Promotion of Innovative R\&D" of the Japan Science and Technology Agency (JST). 


\section{References}

[1] L. Trahms, Colloidal Magnetic Fluids, in: S. Odenbach (Ed.), Springer Berlin Heidelberg, Berlin, Heidelberg, 2009: pp. 327-358.

[2] C.-Y. Hong, C.C. Wu, Y.C. Chiu, S.Y. Yang, H.E. Horng, H.C. Yang, Magnetic susceptibility reduction method for magnetically labeled immunoassay, Appl. Phys. Lett. 88 (2006) 212512.

[3] K. Enpuku, T. Tanaka, Y. Tamai, M. Matsuo, AC susceptibility of magnetic markers in suspension for liquid phase immunoassay, J. Magn. Magn. Mater. 321 (2009) 1621-1624.

[4] S.-H. Chung, A. Hoffmann, K. Guslienko, S.D. Bader, C. Liu, B. Kay, et al., Biological sensing with magnetic nanoparticles using Brownian relaxation (invited), J. Appl. Phys. 97 (2005) 10R101.

[5] K. Enpuku, T. Tanaka, T. Matsuda, F. Dang, N. Enomoto, J. Hojo, et al., Properties of magnetic nanoparticles in the Brownian relaxation range for liquid phase immunoassays, $\mathrm{J}$. Appl. Phys. 102 (2007) 054901.

[6] D. Eberbeck, C. Bergemann, S. Hartwig, U. Steinhoff, L. Trahms, Binding kinetics of magnetic nanoparticles on latex beads and yeast cells studied by magnetorelaxometry, J. Magn. Magn. Mater. 289 (2005) 435-438.

[7] N.L. Adolphi, D.L. Huber, J.E. Jaetao, H.C. Bryant, D.M. Lovato, D.L. Fegan, et al., Characterization of magnetite nanoparticles for SQUID-relaxometry and magnetic needle biopsy., J. Magn. Magn. Mater. 321 (2009) 1459-1464.

[8] A. Tsukamoto, K. Saitoh, D. Suzuki, N. Sugita, Y. Seki, A. Kandori, et al., Development of Multisample Biological Immunoassay System Using HTS SQUID and Magnetic Nanoparticles, IEEE Trans. Appl. Supercond. 15 (2005) 656-659.

[9] K. Enpuku, K. Soejima, T. Nishimoto, H. Tokumitsu, H. Kuma, N. Hamasaki, et al., Liquid phase immunoassay utilizing magnetic marker and high $\mathrm{T}$ [sub c] superconducting quantum interference device, J. Appl. Phys. 100 (2006) 054701.

[10] F. Ludwig, E. Heim, S. Mäuselein, D. Eberbeck, M. Schilling, Magnetorelaxometry of magnetic nanoparticles with fluxgate magnetometers for the analysis of biological targets, $\mathrm{J}$. Magn. Magn. Mater. 293 (2005) 690-695.

[11] A. Tsukamoto, K. Saitoh, N. Sugita, H. Kuma, Y. Sugiura, S. Hamaoka, et al., Improvement of sensitivity of multisample biological immunoassay system using HTS SQUID and magnetic nanoparticles, Phys. C Supercond. 445-448 (2006) 975-978. 
[12] A.D. Hibbs, R.E. Sager, S. Kumar, J.E. McArthur, a. L. Singsaas, K.G. Jensen, et al., A SQUID-based ac susceptometer, Rev. Sci. Instrum. 65 (1994) 2644.

[13] K. Tsukada, T. Kiwa, H. Tahara, Highly Sensitive Measurement of Moisture Content Using HTS-SQUID, IEEE Trans. Appl. Supercond. 19 (2009) 878-881.

[14] K. Enpuku, S. Nabekura, Y. Tsuji, S. Okada, M. Matsuo, a. Tsukamoto, et al., Detection of magnetic nanoparticles utilizing cooled normal pickup coil and high Tc SQUID, Phys. C Supercond. 469 (2009) 1634-1637.

[15] B. Gleich, J. Weizenecker, Tomographic imaging using the nonlinear response of magnetic particles., Nature. 435 (2005) 1214-7.

[16] J.B. Weaver, A.M. Rauwerdink, C.R. Sullivan, I. Baker, Frequency distribution of the nanoparticle magnetization in the presence of a static as well as a harmonic magnetic field, Med. Phys. 35 (2008) 1988.

[17] T. Yoshida, N.B. Othman, T. Tsubaki, J. Takamiya, K. Enpuku, Evaluation of Harmonic Signals for the Detection of Magnetic Nanoparticles, IEEE Trans. Magn. 48 (2012) 37883791.

[18] M.M. Saari, K. Sakai, T. Kiwa, K. Tsukada, Optimization of the Detection Technique for a Vibrating-Sample Magnetometer Using High-Tc SQUID, IEEE Trans. Appl. Supercond. 23 (2013) 1600204-1600204.

[19] M.M. Saari, K. Sakai, T. Kiwa, A. Tsukamoto, S. Adachi, K. Tanabe, et al., Development of a Compact Moving-Sample Magnetometer Using High-Tc Superconducting Quantum Interference Device, Jpn. J. Appl. Phys. 51 (2012) 046601.

[20] S. Adachi, K. Hata, T. Sugano, H. Wakana, T. Hato, Y. Tarutani, et al., Preparation of multilayer films for integrated high-Tc SQUIDs with ramp-edge Josephson junctions, Phys. C Supercond. 468 (2008) 1936-1941.

[21] J. Weizenecker, B. Gleich, J. Rahmer, H. Dahnke, J. Borgert, Three-dimensional real-time in vivo magnetic particle imaging., Phys. Med. Biol. 54 (2009) L1-L10.

[22] S. Behrens, Colloidal Magnetic Fluids, in: S. Odenbach (Ed.), Springer Berlin Heidelberg, Berlin, Heidelberg, 2009: pp. 1-82.

[23] R. Chantrell, S. Hoon, B. Tanner, Time-dependent magnetization in fine-particle ferromagnetic systems, J. Magn. Magn. Mater. 38 (1983) 133-141.

[24] K. Enpuku, T. Morishige, T. Mihaya, T. Miyazai, M. Matsuo, S. Haku, et al., Magnetic Nanoparticle Imaging Using Cooled Pickup Coil and Harmonic Signal Detection, Jpn. J. Appl. Phys. 52 (2013) 087001. 


\section{Figure captions}

Fig. 1. Schematic diagram of the developed AC-DC magnetometer with coil arrangements of (a) first-order planar differential coil for static magnetization measurement and (b) first-order axial differential coil for dynamic magnetization measurement.

Fig. 2. Magnetization curves of the diluted iron oxide nanoparticle in solutions of different concentrations.

Fig. 3. Fitted parameters of saturation magnetization $M_{S}$ and $C$ with respect to the concentration of the iron oxide nanoparticles.

Fig. 4. Simulated data of (a) fundamental, (b) second harmonics, and (c) third harmonics, plotted as a function of the bias of the DC magnetic field upon application of an AC magnetic field amplitude of $30 \mathrm{mT}$. The insets show the relation between the harmonic component signals and the concentration at their maximum points.

Fig. 5. Ratio of harmonic components to fundamental component at their maximum points with respect to concentration.

Fig. 6. Measured second and third harmonic signals of the $24-\mu \mathrm{g} / \mathrm{ml}$ iron oxide nanoparticle solution with respect to the different DC and AC magnetic fields.

Fig. 7. Signal intensity of second and third harmonic signals corresponding to DC bias of 20 and 0 $\mathrm{mT}$, respectively, during application of an AC magnetic field amplitude of $30 \mathrm{mT}$. 


\section{Figures}
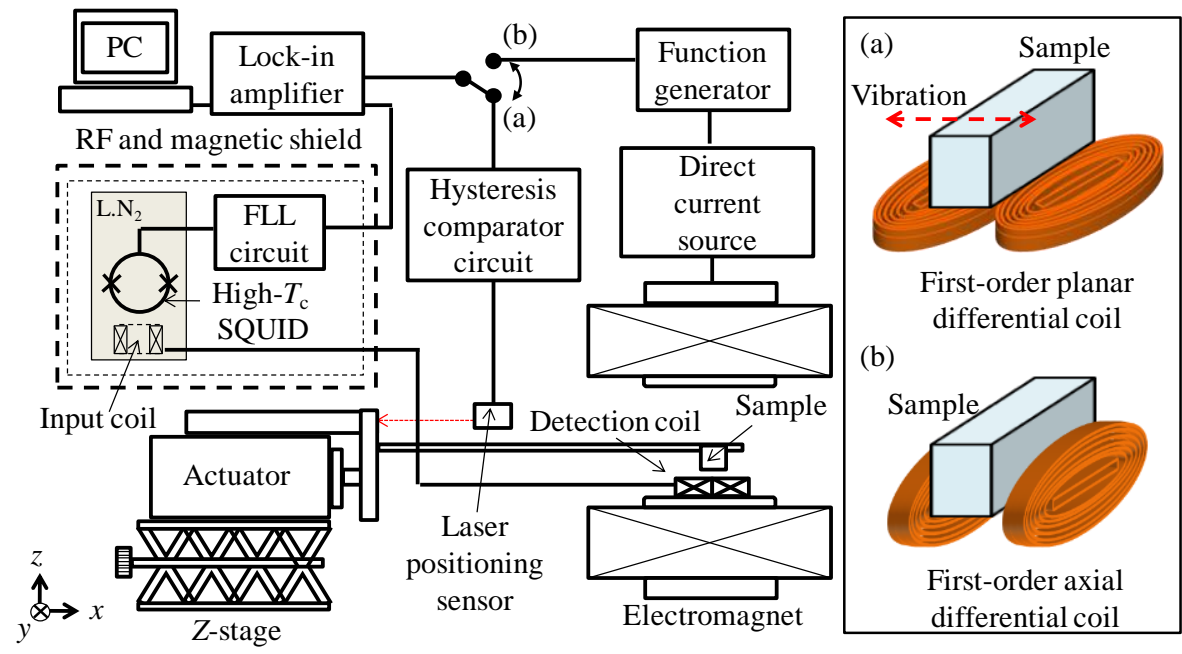

Fig. 1 


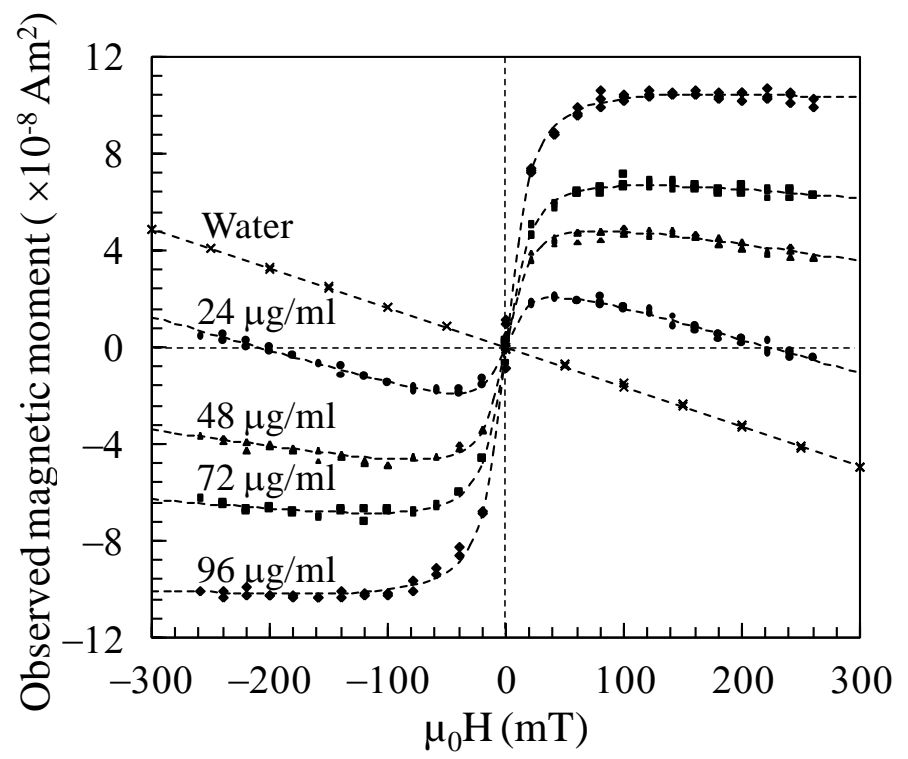

Fig. 2 


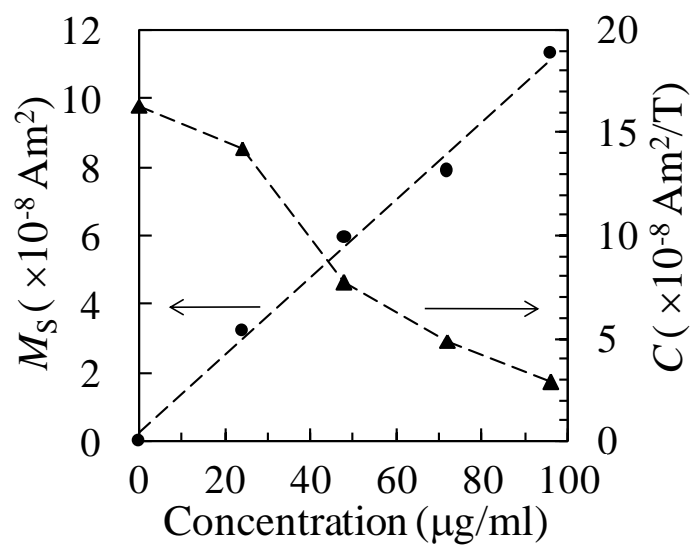

Fig. 3 
(a)

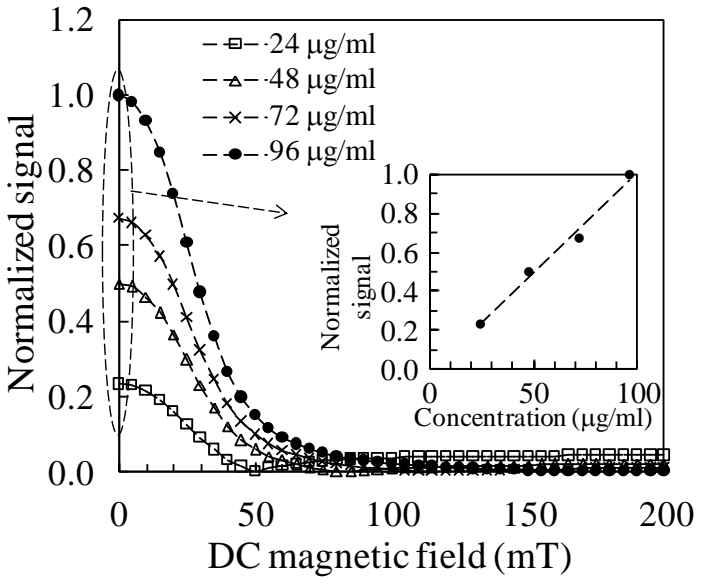

(b)

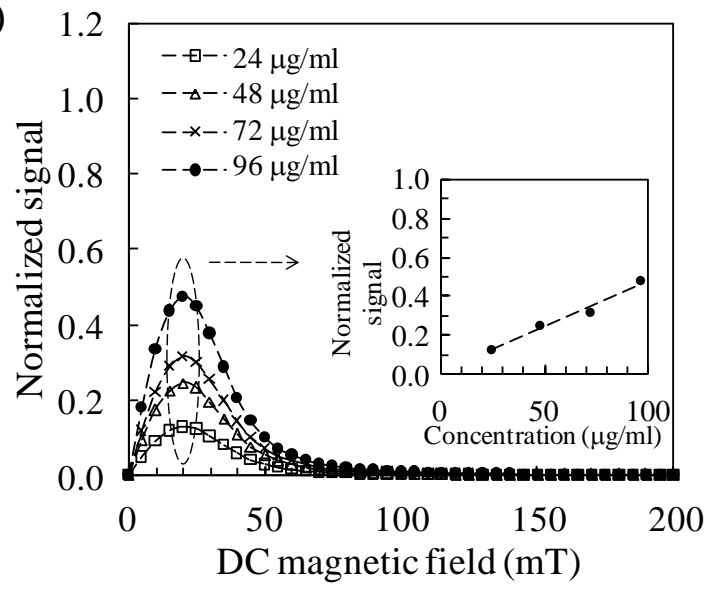

(c)

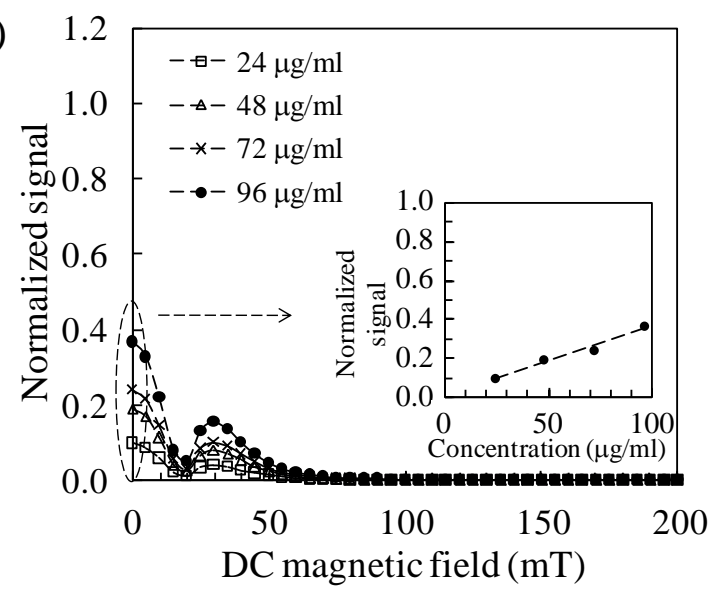

Fig. 4 


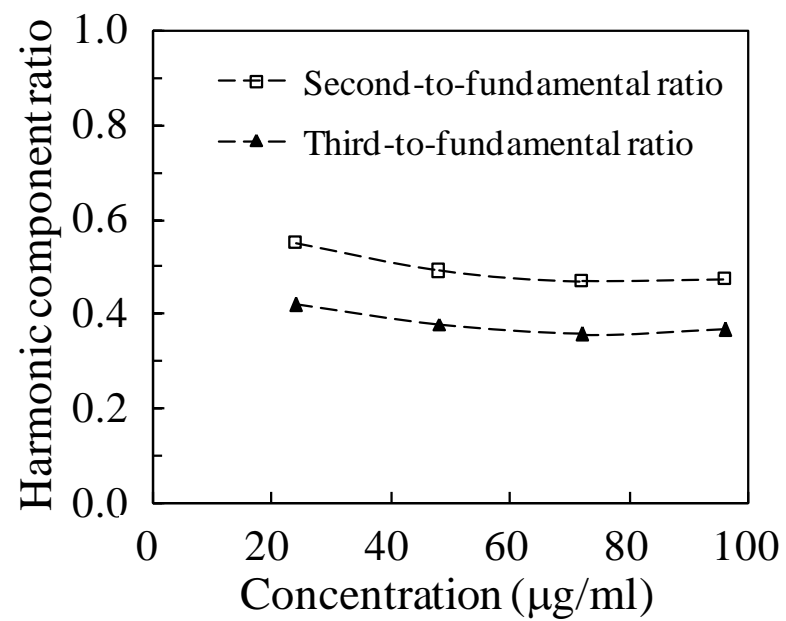

Fig. 5 


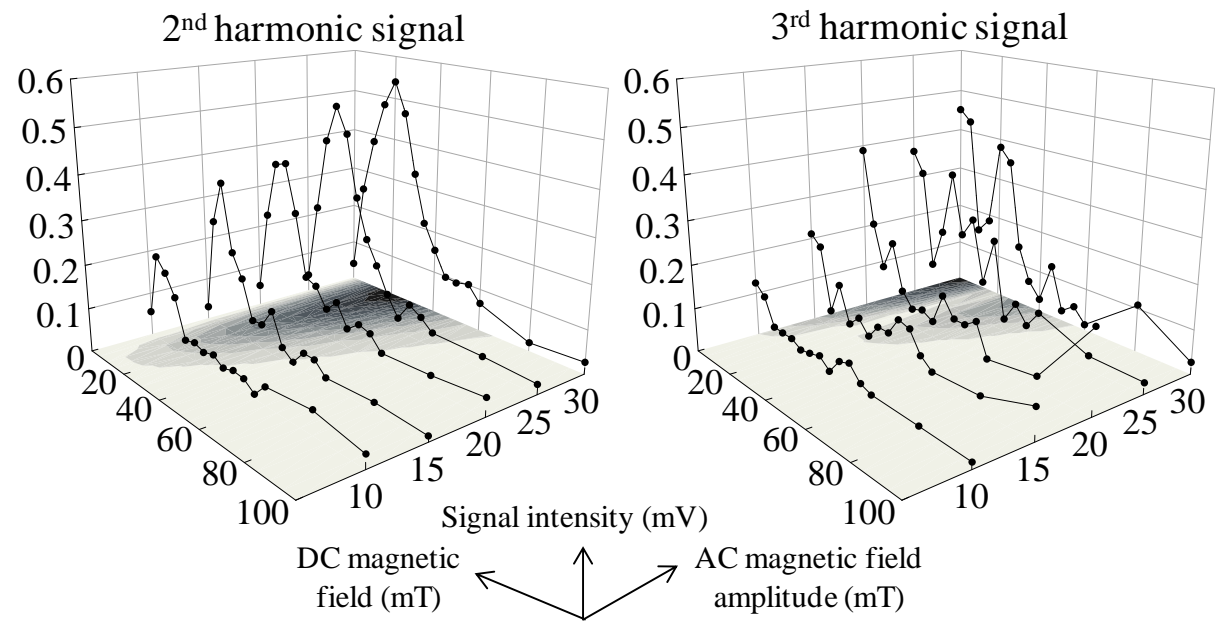

Fig. 6 


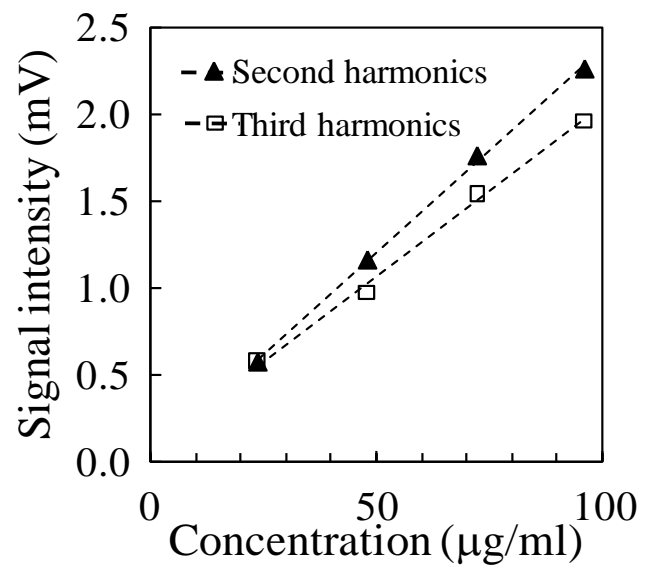

Fig. 7 